\title{
For RF Signal-Based UAV States Recognition, Is Pre- processing Still Important At The Era Of Deep Learning?
}

This paper was downloaded from TechRxiv (https://www.techrxiv.org).

\section{LICENSE}

CC BY 4.0

SUBMISSION DATE / POSTED DATE

$01-11-2021 / 02-11-2021$

\section{CITATION}

Ge, Changhao; Yang, Shubo; Sun, Wenjian; Luo, Yang; Luo, Chunbo (2021): For RF Signal-Based UAV States Recognition, Is Pre-processing Still Important At The Era Of Deep Learning?. TechRxiv. Preprint. https://doi.org/10.36227/techrxiv.16909456.v1

$\mathrm{DOI}$

10.36227/techrxiv.16909456.v1 


\section{For RF Signal-Based UAV States Recognition, Is Pre-processing Still Important At The Era Of Deep Learning?}

\author{
Changhao Ge \\ James Watt School of Engineering \\ University of Glasgow \\ Glasgow, U.K. \\ 2357902G@student.gla.ac.uk
}

Wenjian Sun

School of Information and Communication Engineering

University of Electronic Science and Technology of China

Chengdu, China

sunwenjian@std.uestc.edu.cn

Chunbo Luo

School of Information and Communication Engineering

University of Electronic Science and Technology of China

Chengdu, China

c.luo@uestc.edu.cn

\author{
Shubo Yang \\ Glasgow College \\ University of Electronic Science and Technology of China \\ Chengdu, China \\ 2018190607005@std.uestc.edu.cn
}

Yang Luo

School of Information and Communication Engineering

University of Electronic Science and Technology of China

Chengdu, China

luoyang@uestc.edu.cn

\begin{abstract}
Unmanned Aerial Vehicles (UAVs, also called drones) have been widely deployed in our living environments for a range of applications such as healthcare, agriculture, and logistics. Despite their unprecedented advantages, the increased number of UAVs and their growing threats demand highperformance management and emergency control strategies. To accurately detect a UAV's working state including hovering and flying, data collection from Radio Frequency (RF) signals is a key step of these strategies and has thus attracted significant research interest. Deep neural networks (DNNs) have been applied for UAV state detection and shown promising potentials. While existing work mostly focuses on improving the DNN structures, we discover that RF signals' pre-processing before sending them to the classification model is as important as improving the DNN structures. Experiments on a dataset show that, after applying proposed pre-processing methods, the 10-time average accuracy is improved from $\mathbf{4 6 . 8 \%}$ to $91.9 \%$, achieving nearly $50 \%$ gain comparing with the benchmark work using the same DNN structure. This work also outperforms the state-of-the-art CNN models, confirming the great potentials of data pre-processing for RF-based UAV state detection.
\end{abstract}

Index Terms-UAV, Drone, RF Signal Recognition, Data Preprocessing, Deep Neural Network

\section{INTRODUCTION}

Unmanned Aerial Vehicles (UAV) have been widely used in real-world applications including identifying missing people in a natural disaster [1], providing emergency medical assistance

This research was supported by the National Natural Science Foundation of China under grant 61871096, and National Key R\&D Program of China under grant 2018YFB2101300.
[2], producing maps of forest variables [3], serving as the video source of Augmented Reality (AR) [4], detecting heat leakages [5], crop-dusting [6], and mobile base station [7]. With the increase of UAV's applications, the demands of managing them also rise rapidly. For example, in some no-fly zones, it is vital to detect if there the existence of UAV. Besides existence, the working state of a UAV is also significant, since UAV's working state is key information in scenarios like path optimization and power saving [8], interdicting UAVs operated by malicious entities [9], and building management systems [10] [11]. State recognition has thus become a hot research topic for the efficient management of UAVs.

Existing research on UAV detection has developed a variety of methods. Among them, radar is the mostly used method, while other choices like ambient UAV radio frequency (RF) signals, acoustic sensors, and computer vision techniques [9] are gaining increased attention. Furthermore, they usually detect UAVs' existence instead of the UAV's working state. $\mathrm{RF}$ signals provide the unique opportunity to identify the UAV states because the RF signals of a UAV usually contain rich information about its states. For example, Mhd Saria Allahham et al. [12] proposed an approach that combines RF sensing with Deep Neural Network (DNN) and built a novel UAVs RF dataset, which achieved $46.8 \%$ accuracy for UAV state classification on this dataset. Moreover, convolutional Neural Networks (CNNs) have been applied to the same dataset by Sara Al-Emadi [13] and Mhd Saria Allahham [14], which achieve $59.2 \%$ and $87.4 \%$ in terms of accuracy, respectively. 


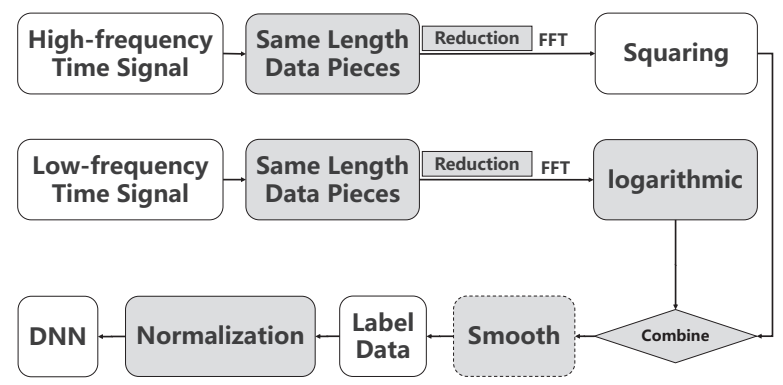

Fig. 1. The overall processing scheme. The gray blocks represent the preprocessing components; the block with dotted border lines represents an optional step.

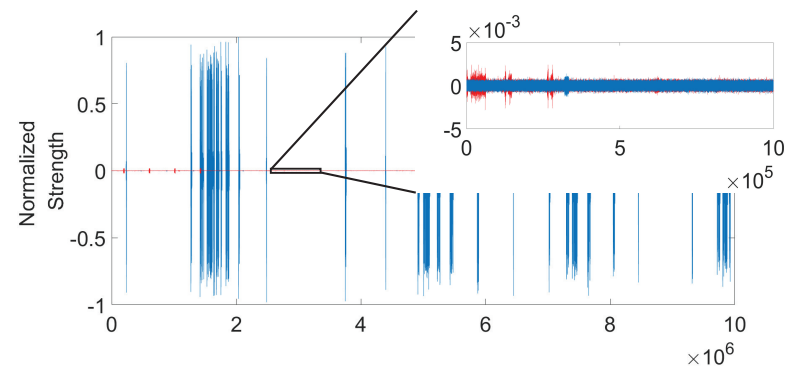

Fig. 2. UAV RF samples. The blue signal is the low-frequency part; the red signal is the high-frequency part. The amplified window displays the noise.

These works concentrate on optimizing the neural networks but they neglect the RF signal processing.

In this paper, we propose to apply four types of methods including data reduction, transformation, cleaning, and integration [15] to process UAV RF signal data before training in a basic DNN. To enable performance comparison, we still use the same open-source dataset of [12] to complete the experiments. The experiments show that our method improves the UAV state recognition accuracy by considerable margins, e.g. from $46.8 \%$ to $91.9 \%$ when using the same DNN. Our method's performance is also superior than CNN based methods, which achieve $59.2 \%$ and $87.4 \%$. The experimental results confirms the importance of data pre-processing methods in the era of deep learning for UAV states recognition.

\section{PROCESSING METHODOLOGY}

This section introduces the data components, network structure, and processing methods. A brief block diagram in Fig. 1 illustrates the whole process.

\section{A. Dataset Components and Network Structure}

The details for the dataset are as follows. The dataset contains a total of 10 types of time-domain signals: one for background noise, and nine for three UAVs in different working states. These signals are composed of two parts: low-frequency and high-frequency parts sampled with 40 $\mathrm{MHz}$ sampling frequency. The low-frequency part contains information of band from $2400 \mathrm{MHz}$ to $2440 \mathrm{MHz}$, while the high-frequency part contains information of band from 2440 $\mathrm{MHz}$ to $2480 \mathrm{MHz}$. The two components are combined after they are converted to the frequency domain by Fast Fourier Transformation (FFT). For the classification model, neural networks are utilized. For a fair comparison, our experiments will use the same DNN as in [12], which has three fully-connected inner layers, with each layer containing 256 neurons.

\section{Algorithm 1 Improved Processing Method \\ Require:}

Input: Low-frequency and High-frequency Time-domain Signals: $\mathbf{l}[n], \mathbf{h}[n]$, where $n$ denotes the $n$th sample value of the sequences; Threshold: $T$; Segment Length: s.

Output: Labled dataset $\mathbf{R}$ of UAV RF signals' frequency magnitude spectra.

\section{Ensure:}

1: Divide $\mathbf{l}[n]$ and $\mathbf{h}[n]$ into $s$ same length segments $\mathbf{l}_{i}[n]$ and $\mathbf{h}_{i}[n]$

2: Initialize $\mathrm{F}$ and $\mathbf{R}$ as empty sequence and matrix.

3: for $i \leq \frac{\operatorname{length}(1[n])}{s}$ do

4: if mean $\left(\mathbf{l}_{i}[n]\right)<T$ and mean $\left(\mathbf{h}_{i}[n]\right)<T$ then

5: $\quad$ Remove both $\mathbf{l}_{i}[n]$ and $\mathbf{h}_{i}[n]$

$$
\text { 6: } \quad \text { else }
$$

7: $\quad$ FFT:

8: $\quad \mathbf{L}_{i}[k]=$ the first half of $\operatorname{abs}\left(f f t\left(l_{i}[n], 2048\right)\right)$

9: $\quad \mathbf{H}_{i}[k]=$ the first half of $\operatorname{abs}\left(\mathrm{fft}\left(h_{i}[n], 2048\right)\right)$

10: $\quad$ where $k$ denotes the $k$ th value of sequences

11: Parallel Transformation:

12: $\quad \mathbf{L}_{i}[k]=\mathbf{L}_{i}^{2}[k]$;

13: $\quad \mathbf{H}_{i}[k]=\lg \left(\mathbf{H}_{i}[k]\right)$

14: Spectra Combination:

15: $\quad \mathbf{H}_{i}[k]=\frac{\operatorname{mean}\left(\mathbf{L}_{i}[1015: 1024]\right)}{\operatorname{mean}\left(H_{i}[1: 10]\right)} \mathbf{H}_{i}[k]$

16: $\quad \mathbf{F}[k]=\left[\mathbf{L}_{i}[k], \mathbf{H}_{i}[k]\right]$

17: $\quad$ Smoothing:

18: $\quad \mathbf{F}[k]=\operatorname{smooth}(\mathbf{F}[k], 5)$

19: $\quad \mathbf{R}=\left[\mathbf{R}, \mathbf{F}^{\prime}\right]$

20: end if

21: end for

22: Label $\mathbf{R}$ with mumber 1 to 10 according to signal type

23: $\mathbf{R}=\mathbf{R}-\min (\min (\mathbf{R}))$

24: $\mathbf{R}=\mathbf{R} / \max (\max (\mathbf{R}))$

25: Return $\mathbf{R}$

\section{B. Preprocessing Methods}

The data pre-processing algorithm is shown in Algorithm 1. We will explain the reasons for applying these methods in this section.

Data Reduction: RF data samples usually include long segments of noise. In Fig. 2, we randomly select a piece of UAV RF data and display noise in an amplified window. Signals like the zoomed-in part in Fig.2 contribute little to classification, and cause confusion to the classification models, leading to reduced recognition accuracy. To only restore the effective RF signals for following classification, the noise data are reduced. 
Data Transformation: This section analyzes and compares the squaring transformation and logarithmic transformation. They are useful to compare the difference between two data samples in the frequency domain. We firstly apply FFT to obtain the frequency-domain signals of any two samples as $\mathbf{F}_{\mathrm{a}}[k]$ and $\mathbf{F}_{\mathrm{b}}[k]$. Then the linear error and squaring error are defined as

$$
\begin{gathered}
\mathbf{d}[k]=\frac{\mathbf{F}_{\mathrm{a}}[k]}{\mathbf{F}_{\mathrm{a}}[k]_{\text {max }}}-\frac{\mathbf{F}_{\mathrm{b}}[k]}{\mathbf{F}_{\mathrm{b}}[k]_{\text {max }}}, \\
\mathbf{D}_{s}[k]=\left(\frac{\mathbf{F}_{\mathrm{a}}[k]}{\mathbf{F}_{\mathrm{a}}[k]_{\max }}\right)^{2}-\left(\frac{\mathbf{F}_{\mathrm{b}}[k]}{\mathbf{F}_{\mathrm{b}}[k]_{\text {max }}}\right)^{2} \\
=\mathbf{d}[k]\left[\frac{\mathbf{F}_{\mathrm{a}}[k]}{\mathbf{F}_{\mathrm{a}}[k]_{\text {max }}}+\frac{\mathbf{F}_{\mathrm{b}}[k]}{\mathbf{F}_{\mathrm{b}}[k]_{\text {max }}}\right] .
\end{gathered}
$$

The footprint $\max$ and $\min$ represent the maximum and minimum values. For a specific $k_{0}$, when the sum of normalized $\mathbf{F}_{\mathrm{a}}\left[k_{0}\right]$ and $\mathbf{F}_{\mathrm{b}}\left[k_{0}\right]$ is greater than 1 , the squaring error is greater than the linear error and vice versa. Thus, squaring enlarges the difference between two large values and reduces the difference between two small values. Most UAVs' RF signals have the characteristics that the power is large in the low-frequency range but small in the high-frequency range. The characteristics can be seen in Fig. 2. Therefore, highfrequency spectra of different types are easy to mix up after normalization.

Logarithmic transformation manifests different characteristics from squaring. To guarantee the tranformation output non-negative, the transformation results should be shifted by substracting its minimum value. Define $F_{A}[k]=\frac{\mathbf{F}_{\mathrm{a}}[k]}{\mathbf{F}_{a}[k]_{\text {min }}}$. Then the output is $\lg \left[\mathbf{F}_{\mathrm{A}}[k]\right], \mathbf{F}_{\mathrm{A}}[k] \in\left[1, \frac{\mathbf{F}_{\mathrm{a}}[k]_{\max }}{\mathbf{F}_{\mathrm{a}}[k]_{\min }}\right]$, and the same for $\mathbf{F}_{\mathrm{B}}[k]$. The $\mathbf{d}[k]$ in Eq. (1) can be modified as $\frac{\mathbf{F}_{\mathrm{A}}[k]}{\mathbf{F}_{\mathrm{A}}[k]_{\max }}-\frac{\mathbf{F}_{\mathrm{B}}[k]}{\mathbf{F}_{\mathrm{B}}[k]_{\text {max }}}$. Then, define logarithmic error as:

$$
\mathbf{D}_{l}[k]=\frac{\lg \left[\mathbf{F}_{\mathrm{A}}[k]\right]}{\lg \left[\mathbf{F}_{\mathrm{A}}[k]_{\max }\right]}-\frac{\lg \left[\mathbf{F}_{\mathrm{B}}[k]\right]}{\lg \left[\mathbf{F}_{\mathrm{B}}[k]_{\max }\right]} .
$$

Because the ratios of maximum and minimum for different signals are in a similar range, ideally assume $\mathbf{F}_{\mathrm{A}}[k]_{\max }=$ $\mathbf{F}_{\mathrm{B}}[k]_{\max }$. Then $\mathbf{D}_{l}[k]-\mathbf{d}[k]$ can be simplified as $P\left(m_{1}\right)-$ $P\left(m_{2}\right)$ where $P(m)$ and its derivative are:

$$
\begin{gathered}
P(m)=\frac{\lg (m)}{\lg \left(m_{\max }\right)}-\frac{m}{m_{\max }}, \\
P^{\prime}(m)=\frac{1}{\lg \left(m_{\max }\right)}\left[\frac{1}{m}-\frac{\lg \left(m_{\max }\right)}{m_{\max }}\right],
\end{gathered}
$$

$m \in\left[1, \mathbf{F}_{\mathrm{A}}[k]_{\max }\right]$. Because $\mathbf{F}_{\mathrm{A}}[k]_{\max } \gg 10, \frac{\lg \left(m_{\max }\right)}{m_{\max }}<1$, and $P^{\prime}(t)$ is a decreasing function, $P(m)$ will increase first and then decrease. If $10>\mathbf{F}_{\mathrm{A}}\left[k_{0}\right]>\mathbf{F}_{\mathrm{B}}\left[k_{0}\right]$, then $\mathbf{D}_{l}\left[k_{0}\right]-$ $\mathbf{d}\left[k_{0}\right]>0$ and if $\mathbf{F}_{\mathrm{A}}\left[k_{0}\right]>\mathbf{F}_{\mathrm{B}}\left[k_{0}\right]>10$, then $\mathbf{D}_{l}\left[k_{0}\right]-$ $\mathbf{d}\left[k_{0}\right]<0$. This means the transformation enlarges difference between small values and reduce it between large values. Since $\mathbf{F}_{\mathrm{A}}[k]_{\max } \gg 10$, the effect of enlarging is greater than that of reduction. Theoretically, logarithmic transformation is a more reasonable choice.
Data Cleaning: Smoothing is a common processing method to reduce the influence from noise or some abnormal fluctuations like a large peak value. Its mathematically-expressed processing is: assuming $s=2 a+1, a \in N$, and $\mathbf{x}[n]$ is a sequence whose length is greater than s. If $X[n]=\operatorname{smooth}(\mathbf{x}[n], s)$, then $\mathbf{X}\left[n_{0}\right]=\left(\frac{1}{s}\right)\left\{\mathbf{x}\left[n_{0}-a\right]+\ldots \mathbf{x}\left[n_{0}\right]+\ldots \mathbf{x}\left[n_{0}+a\right]\right\}$.

Data Integration: For data integration, there are two points needing modification: transformation and combination.

The signal features and characters of both squaring and logarithmic transformations are illustrated. Squaring is more suitable for low-frequency processing while logarithmic operation fits high-frequency processing better. Compared with using a single transformation, it is better to parallelly apply squaring to low-frequency spectra and logarithmic transformation to highfrequency spectra.

Second, to smoothly connect the spectra, a combination method is used in the previous sections, in which the lowfrequency spectrum is adjusted according to the average value of the first ten values of the high-frequency spectrum. However, because the values of the high-frequency spectra are quite small, a tiny variance will be enlarged and transmitted to the low-frequency part. Thus, we turn to adjust the highfrequency part according to the low-frequency average of its last ten values because low-frequency values are much larger and relatively stable than high-frequency values.

\section{EXPERIMENTS AND RESULTS}

We implement the experiments in a successional way. Each experiment is based on previous results. For the DNN training process, we adopt Adam adaptive gradient descent algorithm.

\section{A. Data Preparation and Reduction}

To prove the data reduction's effectiveness, we divide two groups of the same data into $100 \mathrm{~K}$ length segments. One group performs reduction while the other does not. Specifically, if both corresponding high-frequency and low-frequency segments' absolute value averages are less than the threshold value $T=2.5$, we will remove the segments. The size of the dataset will change when applying reduction and the shorter segments are, the larger dataset is. To research the influence of dataset size, we divide another three data groups into segments with length $50 \mathrm{~K}, 20 \mathrm{~K}$, and $10 \mathrm{~K}$ before reduction. Then all these data groups will go through FFT, squaring, and normalization.

The results are in Table I. When segment length becomes shorter, the dataset size and accuracy increase. They are positively correlated. However, when the reduction segment length is $100 \mathrm{~K}$, and almost half of the data are removed, the accuracy still rises by $9.9 \%$; when the length is $50 \mathrm{~K}$, though the dataset size is approximately the same as the group without reduction, the accuracy increases by $15.9 \%$. These results show that, besides the size of the dataset, the redundant data is indeed a factor influencing this classification. 


\begin{tabular}{cccccc}
\hline $\begin{array}{c}\text { Length of } \\
\text { Segments }\end{array}$ & $\begin{array}{c}\mathbf{1 0 0 K} \text {, No } \\
\text { Reduction }\end{array}$ & $\mathbf{1 0 0 K}$ & $\mathbf{5 0 K}$ & $\mathbf{2 0 K}$ & $\mathbf{1 0 K}$ \\
\hline $\begin{array}{c}\text { Theoretical Size } \\
\text { of Each Fold }\end{array}$ & 2064 & 1169 & 2125 & 4806 & 9084 \\
\hline $\begin{array}{c}\text { Average } \\
\text { Accuracy }\end{array}$ & $46.8 \%$ & $56.7 \%$ & $62.4 \%$ & $68.5 \%$ & $72.6 \%$ \\
\hline
\end{tabular}

TABLE I

ACCURACIES OF APPLYING DATA REDUCTION UNDER DIFFERENT SEGMENT LENGTH AND DATA BASE SIZE.

\section{B. Logarithmic Transformation}

Since squaring is used in Section III.A, here we replace squaring operation with logarithmic transformation, but add a step to shift the logarithmic transformation output to the positive range. All groups' accuracies increase much, which means the logarithmic transformation performs better than squaring. However, unexpected low accuracies appear in two groups. A possible reason for this phenomenon is that the neural network converges to a locally optimal solution easily because logarithmic transformation changes the data's structure. We calculate the results without these unreasonable accuracies and these results are marked with $*$.

\begin{tabular}{cccccc}
\hline $\begin{array}{c}\text { Length of } \\
\text { Segments }\end{array}$ & $\begin{array}{c}\mathbf{1 0 0 K , N o} \\
\text { Reduction }\end{array}$ & $\mathbf{1 0 0 K}$ & $\mathbf{5 0 K}$ & $\mathbf{2 0 K}$ & $\mathbf{1 0 K}$ \\
\hline $\begin{array}{c}\text { Average } \\
\text { Accuracy }\end{array}$ & $72.0 \%^{*}$ & $78.1 \%$ & $83.7 \%$ & $83.8 \%$ & $88.3 \%^{*}$ \\
\hline
\end{tabular}

TABLE II

ACCURACIES OF APPLYING LOGARITHMIC TRANSFORMATION UNDER DIFFERENT SEGMENT LENGTH.

\section{Smoothing}

To research the relation between accuracy and span length of smoothing, we select four data groups in previous sections and apply smoothing to them with different span lengths from 1 to 25. The results are shown in Fig. 3. Except for the no-reduction squaring group, the other groups show little improvement. However, smoothing also has no negative influence on them, so smoothing is an optional choice for this problem. If applied, the recommended span's length is 5 .

\section{Parallel Transformation and Spectra Integration}

After applying parallel transformation with the new combination method, this section shows the complete performance of whole processing. Based on Section III.B's experiments, we implement squaring and logarithmic transformations to lowfrequency and high-frequency components respectively, which are combined as algorithm 1 shows. Then smooth them with a span length of 5 and normalize them like Section III.B. The result is shown in Table III. All groups show a dramatic increase in accuracies, and the 50K length group reaches the best average accuracy of $91.9 \%$. More importantly, the locally optimized solution problem in Section III.B is solved by the parallel transformation integration.

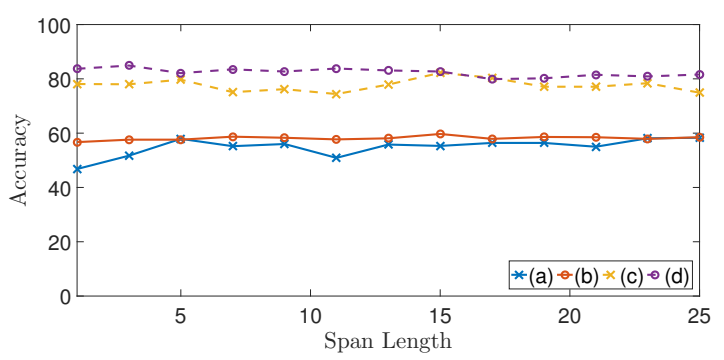

Fig. 3. The results of data groups smoothed with different span lengths. Data groups: (a) 100K, No-reduction, Squaring; (b) 100K, With-reduction, Squaring; (c) 100K, With-reduction, Logarithmic Transformation; (d) 50K, With-reduction, Logarithmic Transformation.

\begin{tabular}{cccccc}
\hline $\begin{array}{c}\text { Length of } \\
\text { Segments }\end{array}$ & $\begin{array}{c}\text { 100K,No } \\
\text { Reduction }\end{array}$ & $\mathbf{1 0 0 K}$ & $\mathbf{5 0 K}$ & $\mathbf{2 0 K}$ & $\mathbf{1 0 K}$ \\
\hline $\begin{array}{c}\text { Average } \\
\text { Accuracy }\end{array}$ & $83.7 \%$ & $87.2 \%$ & $91.9 \%$ & $87.8 \%$ & $88.8 \%$ \\
\hline
\end{tabular}

TABLE III

ACCURACIES WITH THE COMPLETE PROCESSING UNDER DIFFERENT SEGMENT LENGTH.

\section{E. Best Results Comparison}

In summary, $50 \mathrm{~K}$-segment-reduction, squaring-logarithmic parallel transformation, and 5-span-length smoothing contribute to the best approach for the UAV state recognition problem which reaches $91.9 \%$ accuracy with low time-cost and storage consumption. A comparison between existing methods is in Table IV.

\section{CONCLUSION}

In this paper, a data pre-processing approach is proposed to optimize the accuracy of the UAV's working state recognition by using a DNN. We explain the motivation for selecting the data pre-processing methods in redundant data reduction, logarithmic transformation, smoothing, and parallel transformation with proper integration. Then, extensive experiments are carried out to prove their effectiveness. Finally, we achieve a much-enhanced average accuracy of $91.9 \%$ and recommend the best processing approach in this UAV state recognition problem.

\section{REFERENCES}

[1] R. Avanzato, F. Beritelli, and M. Vaccaro, "Identification of mobile terminal with femtocell on drone for civil protection applications," in 2019 10th IEEE International Conference on Intelligent Data Acquisition and Advanced Computing Systems: Technology and Applications (IDAACS), 2019, vol. 1, pp. 269-273.

[2] A. Bitar, A. Jamal, H. Sultan, N. Alkandari, and M. El-Abd, "Medical drones system for amusement parks," in 2017 IEEE/ACS 14th International Conference on Computer Systems and Applications (AICCSA), 2017, pp. 19-20.

[3] J. Wallerman, J. Bohlin, M. B. Nilsson, and J. E. S. Franssen, "Dronebased forest variables mapping of icos tower surroundings," in IGARSS 2018 - 2018 IEEE International Geoscience and Remote Sensing Symposium, 2018, pp. 9003-9006. 


\begin{tabular}{ccccc}
\hline Methods & DNN in [12] & CNN in [13] & CNN in [14] & Data Pre-processing (ours) \\
\hline Best Results & $46.8 \%$ & $59.2 \%$ & $87.4 \%$ & $\mathbf{9 1 . 9 \%}$ \\
\hline
\end{tabular}

TABLE IV

BEST RESULTS OF THE STATE OF THE ART APPROACHES FOR THE UAV STATE RECOGNITION CHALLENGE UNDER DIFFERENT APPROACHES.

[4] M. Unal, E. Bostanci, E. Sertalp, M. S. Guzel, and N. Kanwal, "Geolocation based augmented reality application for cultural heritage using drones," in 2018 2nd International Symposium on Multidisciplinary Studies and Innovative Technologies (ISMSIT), 2018, pp. 1-4.

[5] H. Kayan, R. Eslampanah, F. Yeganli, and M. Askar, "Heat leakage detection and surveiallance using aerial thermography drone," in 2018 26th Signal Processing and Communications Applications Conference (SIU), 2018, pp. 1-4.

[6] D. Yallappa, M. Veerangouda, D. Maski, V. Palled, and M. Bheemanna, "Development and evaluation of drone mounted sprayer for pesticide applications to crops," in 2017 IEEE Global Humanitarian Technology Conference (GHTC), 2017, pp. 1-7.

[7] A. Alsharoa, H. Ghazzai, A. Kadri, and A. E. Kamal, "Spatial and temporal management of cellular hetnets with multiple solar powered drones," IEEE Transactions on Mobile Computing, vol. 19, no. 4, pp. 954-968, 2020.

[8] H. Cho, D. Kim, J. Park, K. Roh, and W. Hwang, "2d barcode detection using images for drone-assisted inventory management," in 2018 15th International Conference on Ubiquitous Robots (UR), 2018, pp. 461465.

[9] İ. Güvenç, O. Ozdemir, Y. Yapici, H. Mehrpouyan, and D. Matolak, "Detection, localization, and tracking of unauthorized uas and jammers," in 2017 IEEE/AIAA 36th Digital Avionics Systems Conference (DASC), 2017, pp. $1-10$

[10] S. Choi, N. Sung, J. Park, I. Ahn, and J. Kim, "Enabling drone as a service: Onem2m-based uav/drone management system," in 2017 Ninth International Conference on Ubiquitous and Future Networks (ICUFN), 2017, pp. 18-20.

[11] J. A. Besada, A. M. Bernardos, L. Bergesio, D. Vaquero, I. Campaña, and J. R. Casar, "Drones-as-a-service: A management architecture to provide mission planning, resource brokerage and operation support for fleets of drones," in 2019 IEEE International Conference on Pervasive Computing and Communications Workshops (PerCom Workshops), 2019, pp. 931-936.

[12] M. F. Al-Sa'd, A. Al-Ali, A.Mohamed, T. Khattab, and A. Erbad, "Rfbased drone detection and identification using deep learning approaches: An initiative towards a large open source drone database," Future Generation Computer Systems, vol. 100, pp. 86-97, 2019.

[13] S. Al-Emadi and F. Al-Senaid, "Drone detection approach based on radio-frequency using convolutional neural network," in 2020 IEEE International Conference on Informatics, IoT, and Enabling Technologies (ICIoT), 2020, pp. 29-34.

[14] M. S. Allahham, T. Khattab, and A. Mohamed, "Deep learning for rf-based drone detection and identification: A multi-channel 1-d convolutional neural networks approach," in 2020 IEEE International Conference on Informatics, IoT, and Enabling Technologies (ICIoT), 2020, pp. 112-117.

[15] H. Fang, "Data processing method of dimensionless," M.S. thesis, Southwest University Chongqing China, 2019. 